\title{
Correction to: Predictability Classes for Forecasting Clients Behavior by Transactional Data
}

Elizaveta Stavinova, Klavdiya Bochenina, and Petr Chunaev

\section{Correction to: \\ Chapter "Predictability Classes for Forecasting Clients Behavior by Transactional Data" in: M. Paszynski et al. (Eds.): Computational Science - ICCS 2021, LNCS 12744, https://doi.org/10.1007/978-3-030-77967-2_16}

The chapter was inadvertently published with incomplete funding information in the acknowledgment. The missing funding information is now added and the chapter has been updated with the changes. 